\title{
PSO-Based Integral Sliding Mode Controller for Optimal Swing-Up and Stabilization of the Cart- Inverted Pendulum System
}

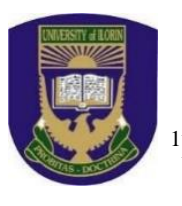

\author{
T. J. Shima ${ }^{1 *}$, H. A. Bashir ${ }^{2}$
}

${ }^{1}$ Department of Electrical \& Electronics Engineering Technology, Harry Pass Polytechnic, Gboko, Nigeria. ${ }^{2}$ Department of Electrical Engineering, Bayero University, Kano, Nigeria.

ABSTRACT: An integral sliding mode controller (ISMC) which employs particle swarm optimization (PSO) algorithm to search for optimal values of the parameters of the integral sliding manifold as well as the gains of the controller is proposed in this work. We considered the swing-up and stabilization of the cart-inverted pendulum system which is assumed to be affected by uncertainties. First, we determined the swing-up and stabilization conditions of the control system by using the internal dynamics of the cart-inverted pendulum system and sliding mode dynamics. A PSO algorithm is then used to search for the optimal values of the ISMC design parameters that satisfy the stabilization condition with the aim of improving the transient performance of the control system. To mitigate the chattering phenomenon, a saturation function of the integral sliding variable was used in the discontinuous control law. Simulation results on swing-up and stabilization of the cart-inverted pendulum system revealed improvement in transient behaviour by reducing settling time (by 52.61\%), overshoots (by 45.56\%) and required track length for cart movement (by 68.34\%).

KEYWORDS: Particle swarm optimization, integral sliding mode, uncertain systems, cart-inverted pendulum, chattering mitigation.

[Received October 20, 2020; Revised Feb. 1, 2021, Accepted June 1, 2021]

Print ISSN: 0189-9546 | Online ISSN: 2437-2110

\section{INTRODUCTION}

Generally, the goal of control system design is to provide a control effort that will yield the desired system response for a given reference input under specified conditions. In practice, however, many practical control systems of high interest are affected by uncertainties, such as external disturbances and unknown plant parameters (Hamayum et al., 2016). For example, launching a rocket into space, stabilization of aircraft in a turbulent airflow, stabilization of a legged mobile robot, are all prone to air resistance. Designing a controller that will give the desired performance for this class of systems is a challenging task, since their accurate mathematical description is difficult to obtain. This paper proposes an improved robust controller in which the parameters of the integral sliding mode controller (ISMC) are optimized using particle swarm optimization (PSO), for one of such uncertain systems - the cart-inverted pendulum system (Krafes et al., 2018).

The cart-inverted pendulum (CIP) system is a mechanical system in which a pendulum is hinged to the centre of a cart via a pivot (Figure 1). When the pendulum is vertically downward, the cart-pendulum system is said to be in its stable equilibrium position (Udhayakumar and Lakshmi, 2007). This position is often referred to as the pendant position. However, when the pendulum is in the upper plane above the cart, the cart-inverted pendulum system becomes unstable (Figure 2). Therefore, given a cart-pendulum system with the pendulum *Corresponding author: shimajt@gmail.com initially in its pendant position, the control task is to provide a suitable control effort that will, first, destabilize the cartpendulum system from its stable equilibrium and swing the pendulum to the upper plane above the cart. Then, it should stabilize the pendulum at the unstable equilibrium point and also return the cart to a reference position on the track.

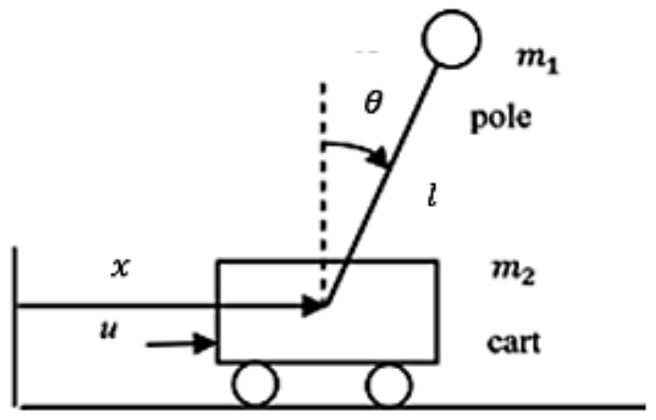

Figure 1: Physical model of the CIP system (Yih, 2013).

Although the cart-inverted pendulum system is seemingly simple from the construction point of view, its control is very challenging. This is because in addition to the effects of external disturbances (e.g. air resistance and uncertainties due to friction) on the open-loop unstable system and its nonlinear nature, the system is under-actuated (Krafes et al., 2018).

doi: http://dx.doi.org/10.4314/njtd.v18i2.2 
The analysis and control of the cart-inverted pendulum system has been considered as a benchmark in nonlinear control and it has received a lot of attention from many researchers in this field. Therefore, many research on control of the mechanical system have been reported in literature.

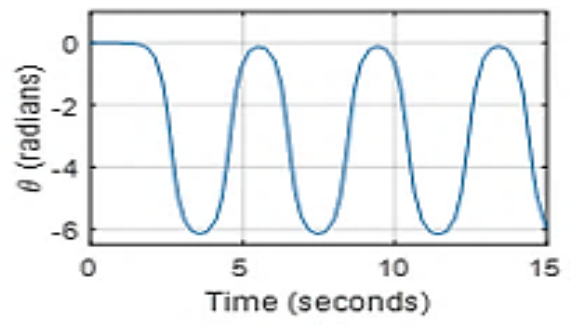

(a)

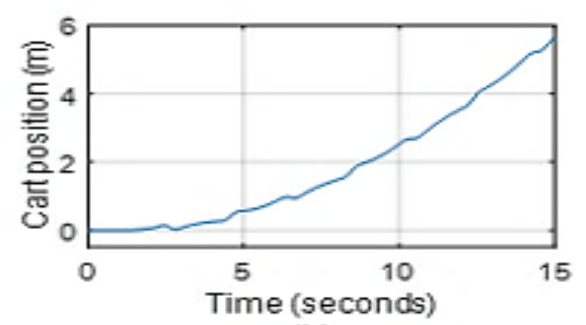

(b)

Figure 2: Dynamics of the CIP system $(\theta(0)=0$ rads $)$.

Energy-based control schemes (Åström and Furuta, 2000; Udhayakumar and Lakshmi, 2007; Kennedy and Tran, 2016) have been proposed for swing-up control of the cart-inverted pendulum system. However, energy-based control of the inverted pendulum generally has two problems (Otani et al., 2001). First, if the pendulum receives more energy than its potential energy at the upright position, then it will pass the position and will not stay at the unstable equilibrium point. Another problem is that the energy-based approach does not control the position of the pivot; it only controls the angle of the pendulum.

Linear control design techniques (Jose et al., 2015; Siradjuddin et al., 2018) have also been proposed for the control of the cart-inverted pendulum system. Linear control algorithms are, however, incapable of handling nonlinear systems where uncertainties and external disturbances are inherently present (Irfan et al., 2018).

Another category of control schemes proposed for the cart-inverted pendulum system is the intelligent control (ElNagar et al., 2013; Yusuf and Magaji, 2014; Al-Mahturi et al., 2019). However, while intelligent control schemes generally offer good performance for a wide range of uncertainties, their implementation requires some good computation power and intelligence (Duarte-Galvan et al., 2012).

Hybrid control schemes (Urniežius and Gegužis, 2014; Patel and Borkar, 2017; Isa et al., 2019; Mahmoodabadi and Haghbayan, 2019) have also been proposed for the cartinverted pendulum system. Such schemes often involve a nonlinear control for swing-up and a linear control for stabilization.
Finally, researchers have also proposed sliding mode control schemes (Yih, 2013; Irfan et al., 2018; Al-Araji, 2019). The advantage of sliding mode controllers is that they are simple to implement and they provide robust performance against uncertainties (Wang et al., 2013).

The conventional SMC possesses a reaching phase during which the system's response is affected by uncertainties. To eliminate the reaching phase and, thereby, ensure insensitivity of the system to uncertainties from the beginning and throughout the entire system's response, the integral sliding mode control (ISMC) scheme was proposed (Edwards et al., 2014).

Although ISMC schemes are simple to design, the analytical techniques for designing them is time-consuming and may not yield optimal design variables especially when dealing with uncertain and nonlinear control systems. Consequently, there is a need to employ the nature-inspired metaheuristic techniques (Okwu and Tartibu, 2021) to obtain optimal design parameters for the ISMC schemes. Among metaheuristic algorithms, PSO is gaining a lot of attention in the control community because of its simplicity, ease of implementation, and fast convergence in finding high-quality solutions (Bejarbaneh et al., 2018).

This paper proposes a PSO-based integral sliding mode control (PSO-ISMC) scheme for the cart-inverted pendulum system. The aim is to employ a PSO algorithm to search for the optimal design parameters of the ISMC to improve the transient performance of the control system while still retaining its robustness property. In this design, the nonlinear dynamic models of the cart-inverted pendulum system are used. The approach presented by Yih (2013) for design of sliding surfaces is adopted in this work. By using a switch, two identical integral sliding mode controllers with different sets of sliding surface parameters are used; one for swing-up control and the other for stabilization. Optimal values of the parameters of the sliding surface are obtained using particle swarm optimization (PSO) technique. PSO requires a fitness function. In this paper, the objective function is a minimizing function which is a linear combination of the integral square error (ISE), integral absolute error (IAE), integral time square error (ITSE), and integral time absolute error (ITAE) performance indices, using appropriate weighting factors.

The rest of the paper is organized as follows: Section II presents the model of the cart-inverted pendulum system and details the design of the control technique under study while Section III discusses the results obtained via simulations. Finally, Section IV concludes the paper.

\section{METHODOLOGY}

\section{A. Dynamic Model of the Cart-Inverted Pendulum System}

The dynamics of the cart-inverted pendulum system (Figure 1) can be described using Euler-Lagrange equations of motion (Siradjuddin et al., 2017). Let $x_{1}=\theta, x_{2}=x, x_{3}=$ $\dot{x}_{1}$, and $x_{4}=\dot{x}_{2}$, then in state-space the model of the cartinverted pendulum system is: 


$$
\begin{gathered}
{\left[\begin{array}{l}
\dot{x}_{1} \\
\dot{x}_{2} \\
\dot{x}_{3} \\
\dot{x}_{4}
\end{array}\right]=\left[\begin{array}{l}
x_{3} \\
x_{4} \\
f_{1} \\
f_{2}
\end{array}\right]+\left[\begin{array}{c}
0 \\
0 \\
g_{1} \\
g_{2}
\end{array}\right] u(t)} \\
y=\left[\begin{array}{llll}
1 & 0 & 0 & 0 \\
0 & 1 & 0 & 0
\end{array}\right]\left[\begin{array}{l}
x_{1} \\
x_{2} \\
x_{3} \\
x_{4}
\end{array}\right]
\end{gathered}
$$

where:

$$
\begin{gathered}
f_{1}=\frac{1}{\Delta}\left(b c g \sin x_{1}+c \mu x_{4} \cos x_{1}-c w \cos x_{1}\right. \\
\left.-0.5 c^{2} x_{3}{ }^{2} \sin 2 x_{1}\right) \\
f_{2}=\frac{1}{\Delta}\left(a c x_{3}^{2} \sin x_{1}+a w-a \mu x_{4}-0.5 c^{2} g \sin 2 x_{1}\right) \\
g_{1}=-\frac{1}{\Delta}\left(c \cos x_{1}\right) \\
g_{2}=\frac{1}{\Delta}(a) \\
a=m_{1} l^{2}+J, b=m_{2}+m_{1}, c=m_{1} l \text { and } \Delta=a b- \\
c^{2} \cos ^{2} x_{1} .
\end{gathered}
$$

Note that $u(t)$ is the control signal applied to the cart, $\mu \dot{x}$ represent frictional forces between the wheels of the cart and the track (where $\mu(\mathrm{Ns} / \mathrm{m}$ ) is the coefficient of viscous friction on the wheels of the cart), and $w$ represents external disturbances.

Equations (1a) and (1b) are in the form of $\dot{\boldsymbol{x}}=\boldsymbol{f}(\boldsymbol{x}, t)+$ $\boldsymbol{g}(\boldsymbol{x}, t) \boldsymbol{u}, \boldsymbol{y}=\boldsymbol{c} \boldsymbol{x}$ where $\boldsymbol{x}=\left[\begin{array}{llll}x_{1} & x_{2} & x_{3} & x_{4}\end{array}\right]^{T}$ is the state vector, $\boldsymbol{u}$ is the control input, $\boldsymbol{f}$ is a nonlinear function of the state variables (including uncertainties and external disturbances $\tilde{\boldsymbol{f}}), \boldsymbol{g}$ is the nonlinear input matrix, $\boldsymbol{y}$ is the output and $\boldsymbol{c}$ is the output matrix. The pendant position of the cartinverted pendulum system is $\left(x_{1}, x_{2}, x_{3}, x_{4}\right)=$ $\left(\pi, x_{2 d}, 0,0\right)$ while the unstable equilibrium point is $\left(x_{1}, x_{2}, x_{3}, x_{4}\right)=\left(0, x_{2 d}, 0,0\right)$ where $x_{2 d}$ is the desired position of the cart.

\section{B. Integral Sliding Mode Control Design}

Sliding mode control (SMC) theory aims at forcing the state trajectories of the plant to converge to a predefined sliding surface and "slide" along the sliding surface until the equilibrium point is reached, by applying a discontinuous control signal (Shtessel et al., 2014). The structure of the sliding mode control scheme is made up of two components, and may be expressed as $u=u_{e q}+u_{s w}$, where $u_{s w}$ is the switching control signal which drives the trajectory of the system to a carefully designed sliding surface, $\left(\sigma_{0}=0\right)$, and $u_{e q}$ is the so-called equivalent control action (Soon et al., 2017) which ensures that the system trajectory stays on the sliding surface after reaching it. The switching function, $\sigma_{0}$, is generally defined as:

$$
\sigma_{0}=\left(\frac{d}{d t}+\lambda\right)^{n-1} e
$$

where $n$ is the order of the system, $\lambda$ is a positive constant which determines the slope of the sliding surface, and $e$ is the tracking error signal.The main feature of this approach is that once the plant's states reach the sliding surface, the system becomes immune to external disturbances (Zhang et al., 2019). However, during the reaching phase of the conventional sliding mode control, invariance of the system's response to external disturbances is not guaranteed (Pan et al., 2017).

To eliminate the reaching phase of the traditional sliding mode control and ensure that the system is immune to external disturbances from the beginning of its response, integral sliding mode control (ISMC) scheme (Hamayum et al., 2016) has been proposed. ISMC assumes that a nominal plant exists, for which a state feedback control $u_{0}$ has already been designed to ensure asymptotic stability of the closed-loop system. A discontinuous control $u_{1}$ is then "added" to the nominal state feedback control to ensure that, in addition to the nominal performance, bounded uncertainties are compensated for without a reaching phase. The structure of the ISMC may, therefore, be expressed as:

$$
u=u_{0}+u_{1}
$$

In ISMC, the switching surface is chosen as in Eq. (4).

$$
\sigma=\sigma_{0}+z
$$

where $\sigma_{0}$ is the conventional sliding manifold given in (2), and $z$ introduces the integral term in the sliding surface.

Let $e_{1}=x_{1}-x_{1 d}$ be the error in the angle of the pendulum and let $e_{2}=x_{2}-x_{2 d}$ be the error in the position of the cart, where $x_{1 d}$ and $x_{2 d}$ are the desired performances of the system. The cart-inverted pendulum system may be viewed as a combination of two second-order subsystems with the states $\left(x_{1}, x_{3}\right)$ and $\left(x_{2}, x_{4}\right)$. Then, we can define two time-varying sliding surfaces $\sigma_{1}=\dot{e}_{1}+\lambda_{1} e_{1}$ and $\sigma_{2}=\dot{e}_{2}+\left(\lambda_{2} / \lambda_{3}\right) e_{2}$, where $\lambda_{1}, \lambda_{2}, \lambda_{3} \in \mathbb{R}^{+}$.

If we choose the desired position of the cart as $x_{2 d}=0$ so that the unstable equilibrium position becomes $(0,0,0,0)$ and if we choose $\lambda_{3}$ as a coupling parameter (Yih, 2013) for combining $\sigma_{1}$ and $\sigma_{2}$, then we can propose the conventional sliding surface as presented in Eq. (5).

$$
\sigma_{0}=\sigma_{1}+\lambda_{3} \sigma_{2}=x_{3}+\lambda_{1} x_{1}+\lambda_{3} x_{4}+\lambda_{2} x_{2}
$$

Hence, the integral sliding manifold in (4) becomes:

$$
\sigma=x_{3}+\lambda_{1} x_{1}+\lambda_{3} x_{4}+\lambda_{2} x_{2}+z
$$

Taking the time derivative of (6) yields:

$$
\dot{\sigma}=\dot{x}_{3}+\lambda_{1} \dot{x}_{1}+\lambda_{3} \dot{x}_{4}+\lambda_{2} \dot{x}_{2}+\dot{z}
$$

Substituting (1a) and (3) into (7), yields Eq. (8). 


$$
\begin{aligned}
\dot{\sigma}= & f_{1}+\lambda_{3} f_{2}+\lambda_{1} x_{3}+\lambda_{2} x_{4}+\left(g_{1}+\lambda_{3} g_{2}\right) u_{0}+ \\
& \left(g_{1}+\lambda_{3} g_{2}\right) u_{1}+\dot{z}
\end{aligned}
$$

During sliding, $\sigma=\dot{\sigma}=0$ and the equivalent control term $u_{1 e q}$ associated with the discontinuous control $u_{1}$ is expected to match the effects of the uncertainties in $f_{1}$ and $f_{2}$. Therefore, we can write

$$
\begin{aligned}
& u_{1 e q}=-\left(\frac{f_{1}+\lambda_{3} f_{2}}{g_{1}+\lambda_{3} g_{2}}\right) \\
& \dot{z}=-\lambda_{1} x_{3}-\lambda_{2} x_{4}-\left(g_{1}+\lambda_{3} g_{2}\right) u_{0}
\end{aligned}
$$

Hence, from (8) we can write

$$
\dot{\sigma}=f_{1}+\lambda_{3} f_{2}+\left(g_{1}+\lambda_{3} g_{2}\right) u_{1}
$$

Define the constant rate reaching law (Khan et al., 2014) as

$$
\dot{\sigma}=-Q \operatorname{sign}(\sigma)
$$

From (11) and (12), we obtain the discontinuous control component as

$$
u_{1}=-\left(\frac{f_{1}+\lambda_{3} f_{2}+Q \operatorname{sign}(\sigma)}{g_{1}+\lambda_{3} g_{2}}\right)
$$

where the constant $Q$ is the modulation gain (Shtessel et al., 2014) which has to be made larger than the size of the uncertainty, and $\operatorname{sign}(\sigma)$ is a signum function of $\sigma$ defined by

$$
\begin{array}{r}
\operatorname{sign}(\sigma)=\left\{\begin{array}{lr}
1, & \text { if } \sigma>0 \\
-1, & \text { if } \sigma<0
\end{array}\right. \\
\operatorname{sign}(0) \in[-1,1]
\end{array}
$$

From (4), we can write

$$
\dot{\sigma}_{0}=\dot{\sigma}-\dot{z}
$$

Therefore, during sliding (i.e. when $\sigma=\dot{\sigma}=0$ ), we can write

$$
\dot{\sigma}_{0}=\lambda_{1} x_{3}+\lambda_{2} x_{4}+\left(g_{1}+\lambda_{3} g_{2}\right) u_{0}
$$

To ensure that $\sigma_{0}$ eventually collapses into $\sigma$, the nominal control action $u_{0}$ that drives the conventional sliding variable $\sigma_{0}$ is selected (Shtessel et al., 2014) as

$$
u_{0}=\frac{-K \sigma_{0}-\lambda_{1} x_{3}-\lambda_{2} x_{4}}{\left(g_{1}+\lambda_{3} g_{2}\right)}
$$

where $K>0$ and is defined as the nominal control gain. Therefore, the structure of the proposed ISMC for the cartinverted pendulum system is obtained as

$$
u=-\left(\frac{K \sigma_{0}+f_{1}+\lambda_{3} f_{2}+\lambda_{1} x_{3}+\lambda_{2} x_{4}+Q \operatorname{sign}(\sigma)}{g_{1}+\lambda_{3} g_{2}}\right)
$$

To ensure a sliding mode from the beginning of the system's response, we set $\sigma(0)=0$ so that from (4) we obtain

$$
z(0)=-\sigma_{0}(0)
$$

Let $\hat{f}_{1}$ and $\hat{f}_{2}$ be the estimates of $f_{1}$ and $f_{2}$ respectively. Then (18) can be rewritten as

$u=-\left(\frac{K \sigma_{0}+\hat{f}_{1}+\lambda_{3} \hat{f}_{2}+\lambda_{1} x_{3}+\lambda_{2} x_{4}+Q \operatorname{sign}(\sigma)}{g_{1}+\lambda_{3} g_{2}}\right)$

where

$$
\hat{f}_{1}=\frac{1}{\Delta}\left(b c g \sin x_{1}+c \mu x_{4} \cos x_{1}-0.5 c^{2} x_{3}{ }^{2} \sin 2 x_{1}\right)
$$

and

$$
\hat{f}_{2}=\frac{1}{\Delta}\left(a c x_{3}^{2} \sin x_{1}-a \mu x_{4}-0.5 c^{2} g \sin 2 x_{1}\right)
$$

To check for the reachability of the control law, we introduce a Lyapunov function of the integral sliding variable as

$$
V=\frac{1}{2} \sigma^{2}
$$

To take the sliding variable to zero in finite time by means of a control $u$, condition (22) has to be achieved.

$$
\dot{V} \leq-\eta|\sigma|
$$

where $\eta$ is a positive real scalar.

The time derivative of (21) yields

$$
\begin{array}{r}
\dot{V}=\sigma \dot{\sigma}=\sigma\left[f_{1}+\lambda_{3} f_{2}+\lambda_{1} x_{3}+\lambda_{2} x_{4}+\left(g_{1}+\right.\right. \\
\left.\left.\lambda_{3} g_{2}\right) u+\dot{z}\right]
\end{array}
$$

If we substitute (10) and (20) into (23) and if we let $\left|f_{1}-\hat{f}_{1}\right| \leq F_{1}$ and $\left|f_{2}-\hat{f}_{2}\right| \leq F_{2}$ then we can write

$$
\dot{V} \leq|\sigma|\left(F_{1}+\lambda_{3} F_{2}\right)-|\sigma| Q
$$

If the modulation gain $Q$ is selected to satisfy the condition:

$$
Q \geq F_{1}+\lambda_{3} F_{2}+\eta
$$

where $\eta>0$ is a real valued scalar, then we have from (24) that

$$
\dot{V} \leq-\eta|\sigma|<0, \sigma \neq 0
$$

Thus, reachability of the control law given in (22) is guaranteed.

\section{1) Design of sliding surface}

The choice of the switching constants $\left(\lambda_{1}, \lambda_{2}\right.$, and $\left.\lambda_{3}\right)$ must be carefully made since the stability of the sliding motion depends on these parameters.

From (1a), we may eliminate the control input so that we can write

$$
g_{2} \dot{x}_{3}-g_{1} \dot{x}_{4}=g_{2} f_{1}-g_{1} f_{2}
$$


From the conventional sliding manifold, the sliding condition is $\dot{\sigma}_{0}=0$. Thus, from Eq. (5) we have

$$
\dot{x}_{3}+\lambda_{3} \dot{x}_{4}=-\lambda_{1} x_{3}-\lambda_{2} x_{4}
$$

Solving Eq. (27) and (28) simultaneously for $\dot{x}_{3}$ and $\dot{x}_{4}$ yields

$$
\begin{aligned}
& \dot{x}_{3}=\frac{g_{1}\left(\lambda_{1} x_{3}+\lambda_{2} x_{4}\right)-\lambda_{3}\left(g_{2} f_{1}-g_{1} f_{2}\right)}{-\left(g_{1}+\lambda_{3} g_{2}\right)} \\
& \dot{x}_{4}=\frac{g_{2}\left(\lambda_{1} x_{3}+\lambda_{2} x_{4}\right)+g_{2} f_{1}-g_{1} f_{2}}{-\left(g_{1}+\lambda_{3} g_{2}\right)}
\end{aligned}
$$

Now, a Jacobian linearization (Perruquetti and Barbot, 2002) of the system of nonlinear equations about the unstable equilibrium position yields

$$
\delta \dot{X}_{1}=A_{1} \delta X_{1}
$$

where the Jacobian matrix $\boldsymbol{A}_{1}$ is obtained as

$$
\boldsymbol{A}_{1}=\left[\begin{array}{cccc}
0 & 0 & 1 & 0 \\
0 & 0 & 0 & 1 \\
\frac{-\lambda_{3} c g}{c-\lambda_{3} a} & 0 & \frac{-c \lambda_{1}}{c-\lambda_{3} a} & \frac{-c \lambda_{2}}{c-\lambda_{3} a} \\
\frac{c g}{c-\lambda_{3} a} & 0 & \frac{a \lambda_{1}}{c-\lambda_{3} a} & \frac{a \lambda_{2}}{c-\lambda_{3} a}
\end{array}\right]
$$

Let $\boldsymbol{I} \in \mathbb{R}^{4 \times 4}$ be an identity matrix and let $s$ be the Laplace variable. Then, the characteristic equation is obtained as

$$
\left|s \boldsymbol{I}-\boldsymbol{A}_{1}\right|=s^{3}+\frac{c \lambda_{1}-a \lambda_{2}}{c-\lambda_{3} a} s^{2}+\frac{c g \lambda_{3}}{c-\lambda_{3} a} s+\frac{c g \lambda_{2}}{c-\lambda_{3} a}=0
$$

Using the Routh-Hurwitz approach (Burns, 2001) the stability conditions are obtained as follows:

$$
\begin{array}{cl}
\text { i. } & \frac{c}{a}>\lambda_{3} \\
\text { ii. } & \frac{\lambda_{2}}{\lambda_{1}}<\frac{c}{a} \\
\text { iii. } & \frac{\lambda_{2}}{\lambda_{1}}<\lambda_{3}
\end{array}
$$

Conditions (i)-(iii) may be effectively combined to yield

$$
\frac{\lambda_{2}}{\lambda_{1}}<\lambda_{3}<\frac{c}{a}
$$

To swing the pendulum from its stable equilibrium position, the cart-pendulum system has to be destabilized to increase the oscillation of the pendulum about the stable equilibrium. Again, the Jacobian linearization of the nonlinear system about the stable equilibrium is

$$
\delta \dot{X}_{2}=A_{2} \delta X_{2}
$$

where the Jacobian matrix $A_{2}$ is found to be

$$
\boldsymbol{A}_{2}=\left[\begin{array}{cccc}
0 & 0 & 1 & 0 \\
0 & 0 & 0 & 1 \\
\frac{-\lambda_{3} c g}{c+\lambda_{3} a} & 0 & \frac{-c \lambda_{1}}{c+\lambda_{3} a} & \frac{-c \lambda_{2}}{c+\lambda_{3} a} \\
\frac{c g}{c+\lambda_{3} a} & 0 & \frac{-a \lambda_{1}}{c+\lambda_{3} a} & \frac{-a \lambda_{2}}{c+\lambda_{3} a}
\end{array}\right]
$$

Thus, the characteristic polynomial equation is

$$
\left|s \boldsymbol{I}-\boldsymbol{A}_{2}\right|=s^{3}+\frac{c \lambda_{1}+a \lambda_{2}}{c+\lambda_{3} a} s^{2}+\frac{c g \lambda_{3}}{c+\lambda_{3} a} s+\frac{c g \lambda_{2}}{c+\lambda_{3} a}=0
$$

Using the Routh-Hurwitz approach, we observe that the system will be destabilized if

$$
\lambda_{3}<\frac{\lambda_{2}}{\lambda_{1}}
$$

The signum function described in (14) causes control chattering and the system may become unstable. One solution to this problem is to approximate the signum function by some smooth function (Shtessel et al., 2014). Thus, a saturation function is chosen to replace the signum function so that the control law in (20) is approximated to

$u=-\left(\frac{K \sigma_{0}+\hat{f}_{1}+\lambda_{3} \hat{f}_{2}+\lambda_{1} x_{3}+\lambda_{2} x_{4}+Q \operatorname{sat}(\sigma)}{g_{1}+\lambda_{3} g_{2}}\right)$

where $\operatorname{sat}(\sigma)$ is a saturation function of the sliding variable $\sigma$ and is described by

$$
\operatorname{sat}(\sigma)= \begin{cases}\sigma, & \text { if }|\sigma| \leq 1 \\ \operatorname{sign}(\sigma), & \text { if }|\sigma|>1\end{cases}
$$

\section{2) Optimization of the ISMC using PSO Algorithm}

In the design of the ISMC (39), any choice of $\lambda_{1}, \lambda_{2}, \lambda_{3}, K, Q \in \mathbb{R}^{+}$(such that $Q$ is greater than the magnitude of the disturbance and $\frac{\lambda_{2}}{\lambda_{1}}<\lambda_{3}<\frac{c}{a}$ ) will guarantee stability of the system. However, optimal selection of these parameters will help to improve the overall system performance. Thus, in this paper, PSO algorithm is used to optimize the ISMC parameters to suit the peculiarities of the cart-inverted pendulum. The objective function in this optimization problem incorporates ISE, IAE, ITSE, and ITAE so that both errors that persist for a long duration and those occurring for a short duration are equally penalized. Thus, the objective function in this optimization problem is formulated as:

$$
\begin{aligned}
F= & \int_{0}^{T}\left(w_{1} e^{2}(t)+w_{2}|e(t)|+w_{3} t e^{2}(t)+\right. \\
& \left.w_{4} t|e(t)|\right) d t
\end{aligned}
$$

where $T$ is the time when the response reaches steady state and $w_{1}, w_{2}, w_{3}, w_{4} \in[0,1]$ are positive real constants which denote the weighting factors. The tracking error in the angle of the pendulum is:

$$
e_{1}(t)=x_{1}-x_{1 d}
$$

and the tracking error in the position of the cart is

$$
e_{2}(t)=x_{2}-x_{2 d}
$$


The optimization objective is to minimize $\mathrm{F}$ subject to $\lambda_{1}>0, \lambda_{2}>0, \lambda_{3}>0, Q>0, K>0$, and $\left(\lambda_{2} / \lambda_{1}\right)<\lambda_{3}<$ $(c / a)$. Specifically, the chaos-based PSO algorithm, proposed by Hong et al.(2016), is used in this work. In this algorithm, the velocity and the position of a particle are updated as in (44) and (45), respectively.

$$
\begin{aligned}
v_{i}(t+1)= & \chi\left[\omega v_{i}(t)+c_{1}\left(P_{\text {best } i}(t)-x_{i}(t)\right)+\right. \\
& \left.c_{2}\left(g_{\text {best }}(t)-x_{i}(t)\right)\right] \\
x_{i}(t+1)= & x_{i}(t)+v_{i}(t+1)
\end{aligned}
$$

where $P_{\text {best }}(t)$ is particle's previous best position, and $g_{\text {best }}$ is the global best position in the swarm. The parameters $\chi, \omega$, $c_{1}$, and $c_{2}$ are the constriction coefficient, the inertia weight, the cognitive coefficient, and the social coefficient, respectively, and are expressed as follows:

$$
\begin{aligned}
& \chi=2 /\left(\phi-2+\sqrt{\phi^{2}-4 \phi}\right) \\
& \omega=0.5 \times \operatorname{rand}(\cdot)+0.5 \times z(t+1) \\
& c_{1}(t)=c_{1 f}-\frac{\mathrm{t}}{\text { maximum iterations }}\left(c_{1 f}-c_{1 i}\right) \\
& c_{2}(t)=c_{2 i}+\frac{\mathrm{t}}{\text { maximum iterations }}\left(c_{2 f}-c_{2 i}\right)
\end{aligned}
$$

where

$$
\phi=c_{1}+c_{2}
$$

and $z$ is the sine chaotic map given by

$$
z(t+1)=\left|\sin \left(\frac{\pi z(t)}{\operatorname{rand}()}\right)\right|
$$

Table 1 shows the values of the parameters used in the algorithm while the algorithm of the PSO variant used is described by the flowchart of Figure 3.

\begin{tabular}{lll}
\multicolumn{2}{l}{ Table 1: PSO } & \\
\hline S/No & Parameters used. & Value used \\
\hline 1. & Cognitive factor $\left(c_{1}\right)$ & 4.5 to 2.5 \\
2. & Social factor $\left(c_{2}\right)$ & 2.5 to 4.5 \\
3. & Maximum iteration & 150 \\
4. & Number of variables & 5 \\
5. & Number of particles & 50 \\
\hline
\end{tabular}

\section{RESULTS AND DISCUSSION}

In this section, the performance of the proposed control scheme on the control of the cart-inverted pendulum system is evaluated and the control scheme is validated by comparing its performance with that of the classical ISMC. The cart-inverted pendulum system whose parameters are shown in Table 2 was considered.

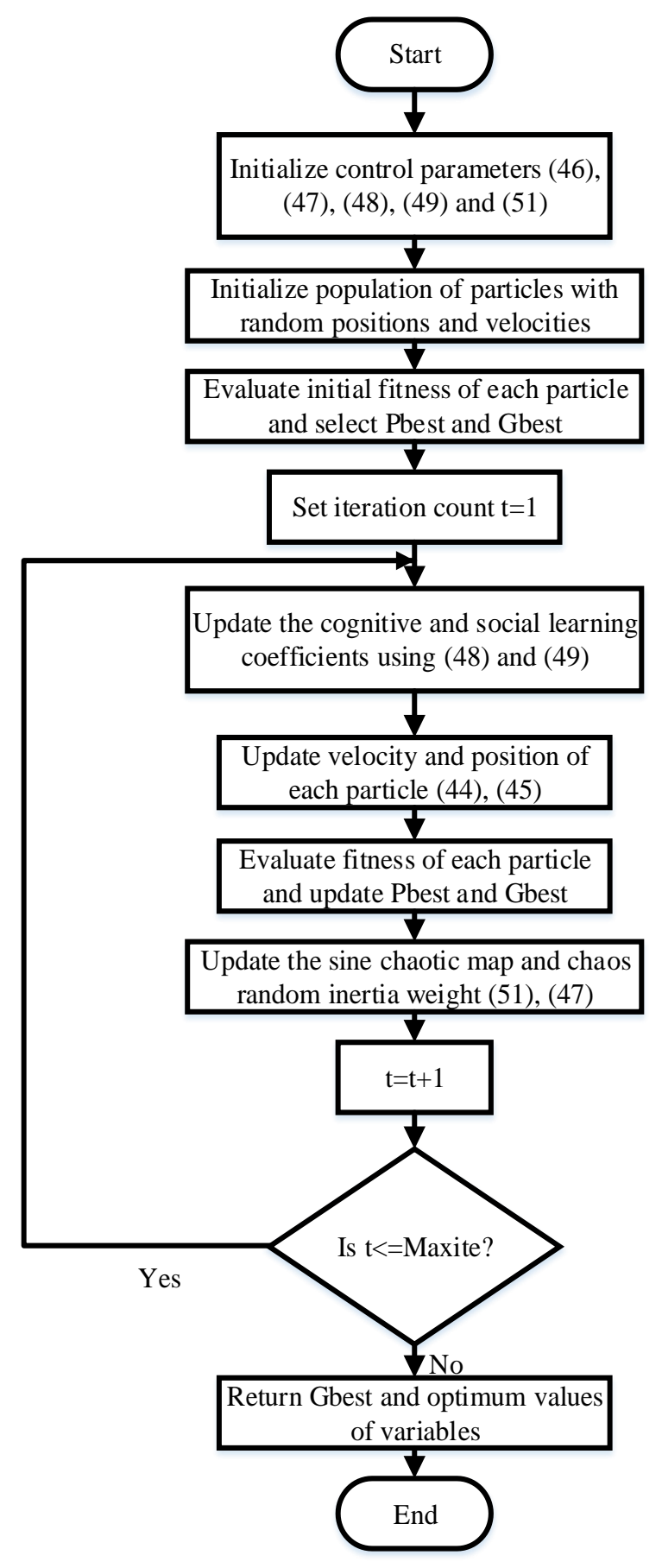

Figure 3: Flowchart of PSO algorithm (Hong et al., 2016).

\section{A. Performance Evaluation of the PSO-ISMC}

The parameters $\lambda_{1}, \lambda_{2}, \lambda_{3}, Q$, and $K$ formed the variables for the optimization. In order to constantly satisfy the stabilization condition in (34), the boundary conditions of the variables are chosen as $\left[\begin{array}{lllll}\lambda_{1 L} & \lambda_{2 L} & \lambda_{3 L} & Q_{L} & K_{L}\end{array}\right]=$ $\left[\begin{array}{lllll}2 & 0.1 & 0.5 & 0 & 0\end{array}\right]$ and $\left[\begin{array}{lllll}\lambda_{1 U} & \lambda_{2 U} & \lambda_{3 U} & Q_{U} & K_{U}\end{array}\right]=$

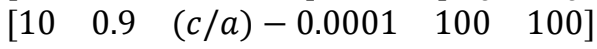


where the subscript $L$ denotes the lower boundary and the subscript $U$ denotes the upper boundary. A maximum of 150 iterations were performed and the PSO algorithm converged to the best fitness function value of 39.24. The convergence characteristic of the PSO algorithm for the first 110 iterations is shown in Figure 4.

Table 2: Parameters of the CIP (Yih, 2013).

\begin{tabular}{|l|c|c|}
\hline Parameter & Symbol & Value \\
\hline Mass of the cart & $m_{2}$ & $12.5 \mathrm{~kg}$ \\
Mass of the pendulum & $m_{1}$ & $2.5 \mathrm{~kg}$ \\
Acceleration of gravity & $g$ & $9.81 \mathrm{~m} / \mathrm{s}$ \\
Coefficient of friction on the wheels & & \\
of the cart & $\mu$ & $0.5 \mathrm{Ns} / \mathrm{m}$ \\
Moment of inertia of the pendulum & $J$ & $0.099 \mathrm{kgm}^{2}$ \\
Length of the pole & $l$ & $0.5 \mathrm{~m}$ \\
\hline
\end{tabular}

For the stabilization of cart-inverted pendulum system, the PSO converged to optimal values $\lambda_{1}=3.8794, \lambda_{2}=$ 0.4191, $\lambda_{3}=0.8288, Q=83.2513$, and $K=99.5374$ for the ISMC parameters while for the swing-up control, $\lambda_{1}=2$, $\lambda_{2}=12$, and $\lambda_{3}=2.5$ was selected for the sliding surface to satisfy condition (38). The disturbance signal $w=$ $2 \sin 50 t N$ was created to test for robustness of the control

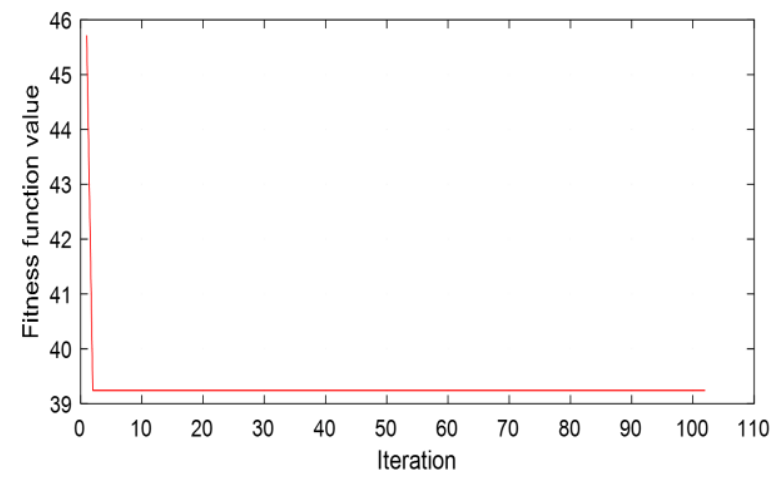

Figure 4: Convergence characteristic of the PSO algorithm.

scheme.

The results of the proposed PSO-ISMC for the control of the cart-inverted pendulum system are shown in Figures 5 and 6. Figure 5(a) illustrates that the proposed control scheme can stabilize the pendulum at $\theta=0$ radians once the pole is found near the unstable equilibrium point. Figure 5(b) shows that the control scheme can simultaneously move the cart to its reference position even in the presence of matched uncertainties. In Figure 5(c), it is observed that a sliding mode is initiated from the beginning of the response of the system.

This ensures insensitivity of the system to uncertainties throughout the response of the control system. The use of the saturation function has also attenuated the chattering phenomenon as shown in Figure 5(d).

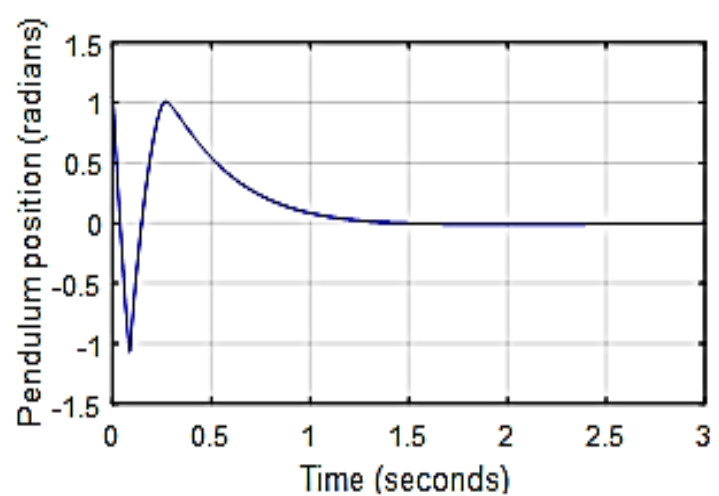

(a)

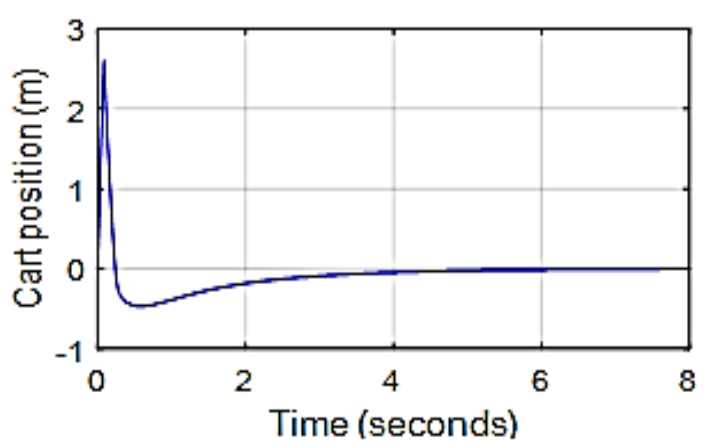

(b)

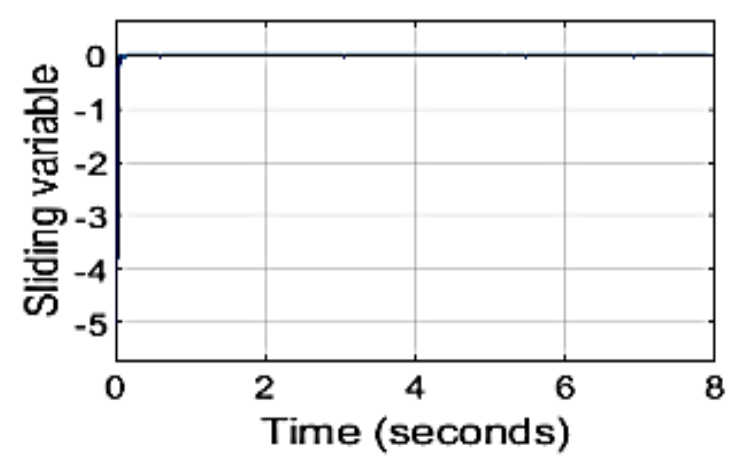

(c)

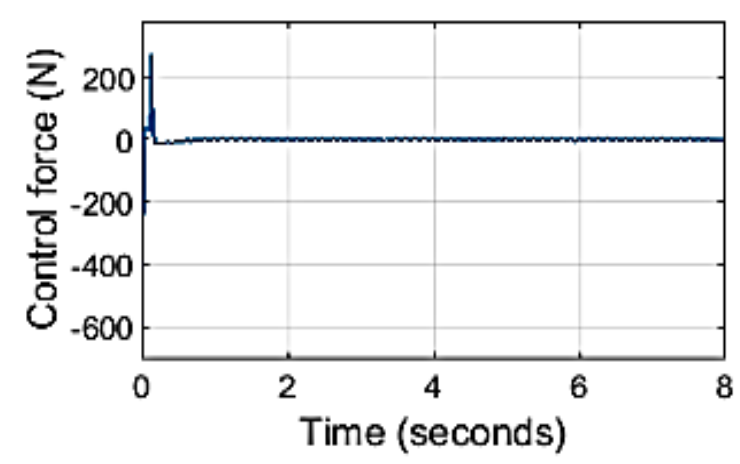

(d)

Figure 5: PSO-ISMC for stabilization of the CIP system $\left(x_{1}(0)=\pi / 3\right.$ rads $)$. 
For swing-up and stabilization of the cart-inverted pendulum system, the two sets of parameters are used so that the controller switches from the swing-up parameters to the stabilization parameters when $\theta \approx 0$ radians and stabilizes the pendulum at the unstable equilibrium position (Figure 6(a)).

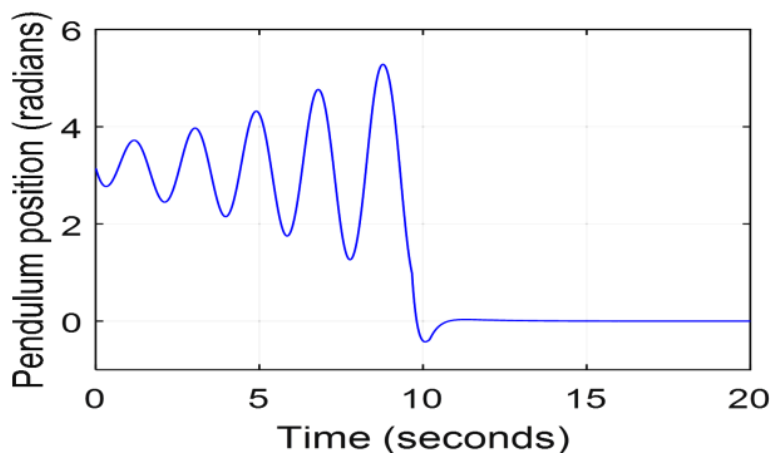

(a)

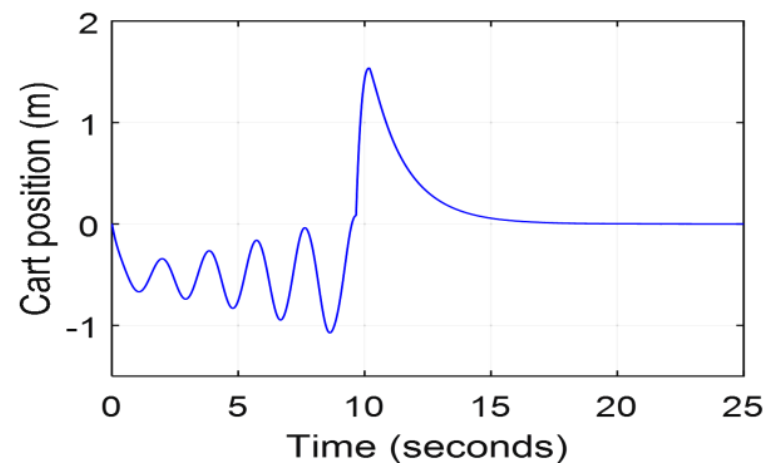

(b)

Figure 6: PSO-ISMC for swing-up and stabilization of the CIP svstem.

Again, Figure 6(b) shows that the cart is returned to its initial position as desired.

\section{B. Validation of the Proposed PSO-ISMC}

To validate the proposed control scheme, the performance of the designed PSO-ISMC is compared with that of the classical ISMC on the control of a chosen cart-inverted pendulum system.

In this comparison, the swing-up parameters $\lambda_{1}=2$, $\lambda_{2}=12$, and $\lambda_{3}=2.5$ are used for the two controllers. For stabilization control, however, the optimal parameters $\lambda_{1}=$ 3.8794, $\lambda_{2}=0.4149, \lambda_{3}=0.8288, Q=83.2513$, and $K=$ 99.5374 are utilized for the PSO-ISMC while the parameters $\lambda_{1}=5, \lambda_{2}=0.5, \lambda_{3}=0.4$ (selected to satisfy the stabilization condition (39)), $Q=5$, and $K=40$ are used for the classical ISMC. The magnitude of the input disturbance is also maintained at $w=2 \sin 50 t N$. Figure 7 and Figure 8 compare the performances of the ISMC and PSO-ISMC on the control of the cart-inverted pendulum system whose parameters are listed in Table 2.

On the stabilization of the pendulum at $\theta=0$ radians (Figure 7(a)), the settling time with the proposed PSO-ISMC is 1.5 seconds while the ISMC achieves the same stabilization task with a settling time of 9.6 seconds. Again, on the control of the cart position (Figure 7(b)), the PSO-ISMC is able to return the cart to its initial position in 4.3 seconds while the ISMC attains same steady-state in 12.1 seconds. Figure 8(a) illustrates the ability of the controllers to swing the pendulum

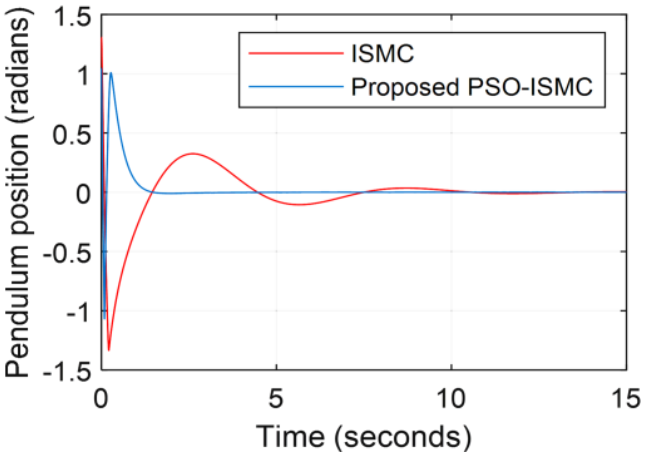

(a)

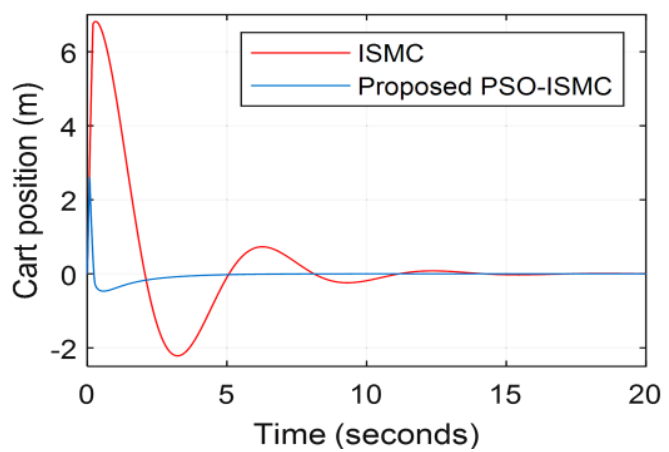

(b)

Figure 7: Stabilization of the cart-inverted pendulum $\operatorname{system}\left(x_{1}(0)=\frac{\pi}{3}\right.$ rads).

up from $\theta=\pi$ radians and stabilize it at $\theta=0$ radians with no steady-state error. However, with the basic ISMC, the maximum track length requirement for the movement of the cart is $8.18 \mathrm{~m}$ whereas the PSO-ISMC requires a maximum track length of $2.59 \mathrm{~m}$ for the movement of the cart as shown in Figure 8(b). This represents $68.34 \%$ reduction in the required maximum length of the track.

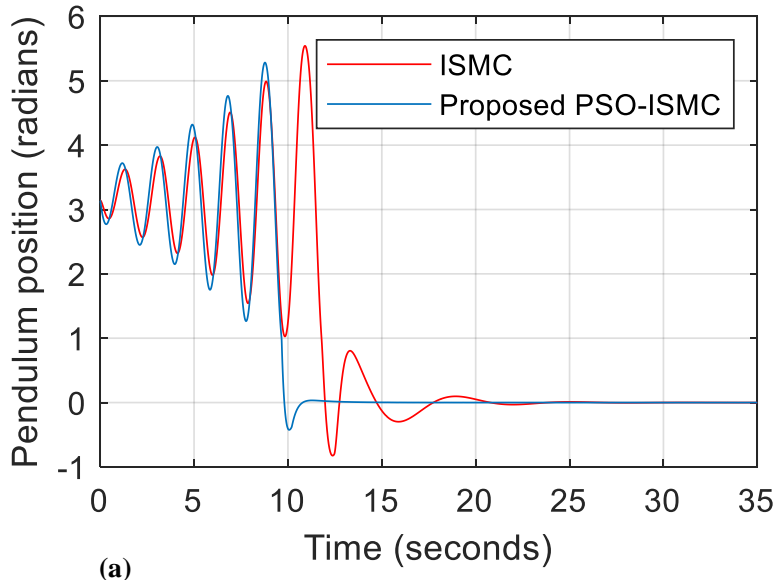




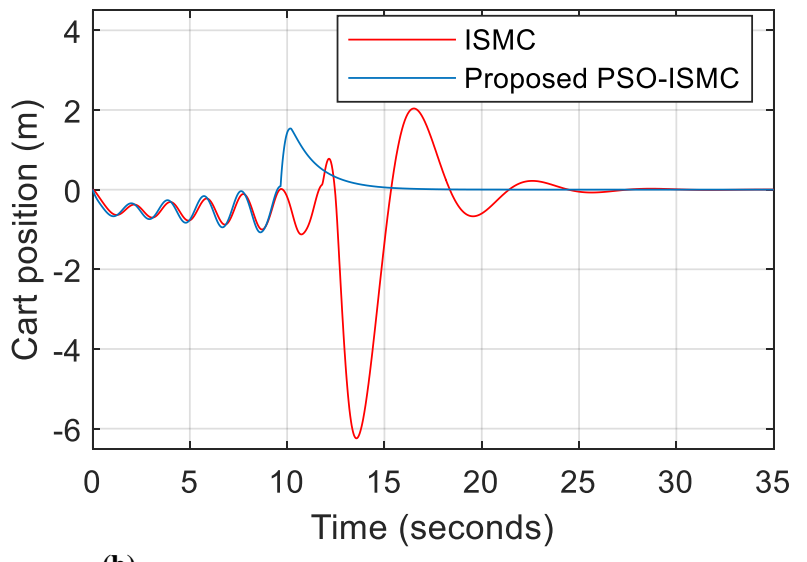

(b)

Figure 8: Swing-up and stabilization of the cart-inverted pendulum system.

\section{CONCLUSION}

This study proposed a PSO-ISMC scheme for swing-up and stabilization of the cart-inverted pendulum system. The swing-up and stabilization conditions of the control system are determined using the internal dynamics of the cart-inverted pendulum system and sliding mode dynamics. Since the integral sliding surface design does not specify unique values for the parameters of the sliding manifold, particle swarm optimization algorithm was used to search for optimal values for the ISMC stabilization parameters. Validation results revealed that the proposed PSO-ISMC scheme yields improvements (in overshoots, settling time and track length requirement) over the conventional ISMC on both the swingup and stabilization of the cart-inverted pendulum system as desired.

\section{REFERENCES}

Al-Araji, A. S. (2019). An adaptive swing-up sliding mode controller design for a real inverted pendulum system based on Culture-Bees algorithm. European Journal of Control, 45(3): 45-56.

Al-Mahturi, A.; F. Santoso; M. A. Garratt and S. G. Anavatti. (2019). An Intelligent Control of an Inverted Pendulum Based on an Adaptive Interval Type-2 Fuzzy Inference System. Paper presented at International Conference on Fuzzy Systems (ICFS2019), New Orleans, USA, 1-6, USA: IEEE.

Åström, K. J. and Furuta, K. (2000). Swinging up a pendulum by energy control. Automatica, 36(2): 287-295.

Bejarbaneh, E. Y.; A. Bagheri; B. Y. Bejarbaneh and S. Buyamin. (2018). Optimization of Model Reference Adaptive Controller for the Inverted Pendulum System Using CCPSO and DE Algorithms. Automatic Control and Computer Sciences, 52(4): 256-267.

Burns, S. R. (2001). Advanced Control Engineering. Butterworth-Heinemann, Woburn, UK.

Duarte-Galvan, C.; I. Torres-Pacheco; R. G. Guevara-Gonzalez; R. J. Romero-Troncoso; L. M. Contreras-Medina; M. A. Rios-Alcaraz and J. R. Millan-
Almaraz. (2012). Review. Advantages and Disadvantages of Control Theories Applied in Greenhouse Climate Control Systems. Spanish Journal of Agricultural Research, 10(4): 926938.

Edwards, C.; H. Alwi and M. T. Hamayun. (2014). Fault tolerant control using integral sliding modes. Paper presented at European Control Conference (ECC2014), Strasbourg, France, 2291-2296: IEEE.

El-Nagar, A. M.; M. El-Bardini and N. M. El-Rabaie. (2013). Intelligent control for nonlinear inverted pendulum based on interval type-2 fuzzy PD controller. Alexandria Engineering Journal, 53(1): 23-32.

Hamayum, M. T.; C. Edwards and H. Alwi. (2016). Fault Tolerant Control Schemes Using Integral Sliding Modes. Studies in Systems, Decision and Control, 61(2): 17-37.

Hong, Y. Y.; A. A. Beltran and A. C. Paglinawan. (2016). A Chaos-Enhanced Particle Swarm Optimization with Adaptive Parameters and Its Application in Maximum Power Point Tracking. Mathematical Problems in Engineering, 2016(1): 1-19.

Irfan, S.; A. Mehmood; M. T. Razzaq and J. Iqbal. (2018). Advanced sliding mode control techniques for Inverted Pendulum: Modelling and simulation. Engineering Science and Technology, an International Journal, 21(4): 753-759.

Isa, A. I.; M. F. Hamza and M. Muhammad. (2019). Hybrid Fuzzy Control of Nonlinear Inverted Pendulum System. Bayero Journal of Engineering and Technology (BJET), 14(2): 200-208.

Jose, A.; C. Augustine; S. M. Malola and K. Chacko. (2015). Performance Study of PID Controller and LQR Technique for Inverted Pendulum. World Journal of Engineering and Technology, 3(2): 76-81.

Kennedy, E. and Tran, H. (2016). Swing-up of an Inverted Pendulum on a Cart Using a Modified Energy Based Approach. Proceedings of the International MultiConference of Engineers and Computer Scientists (IMECS), Hong Kong, China, 6-11.

Khan, Q.; A. I. Bhatti and A. Ferrara. (2014). Dynamic Sliding Mode Control Design Based on an Integral Manifold for Nonlinear Uncertain Systems. Journal of Nonlinear Dynamics, 2014(1): 1-10.

Krafes, S.; Z. Chalh and A. Saka. (2018). A Review on the Control of Second Order Underactuated Mechanical Systems. Complexity, 2018(1): 1-17.

Mahmoodabadi, M. J. and Haghbayan, H. K. (2019). An optimal adaptive hybrid controller for a fourth-order underactuated nonlinear inverted pendulum system. Transactions of the Institute of Measurement and Control, 42(2): 285-294.

Okwu, M. O. and Tartibu, L. K. (2021). Metaheuristic Optimization: Nature-Inspired Algorithms Swarm and Computational Intelligence, Theory and Applications. Studies in Computational Intelligence, 927.

Otani, Y.; T. Kurokami; A. Inoue and Y. Hirashima. (2001). A Swing-Up Control of an Inverted Pendulum with Cart Position Control. Paper presented at Conference on New Technologies for Computer Control (NTCC 2001). Hong Kong, China, 395-400: IFAC. 
Pan, Y.; C. Yang; L. Pan and H. Yu. (2017). Integral Sliding Mode Control: Performance, Modification, and Improvement. IEEE Transactions on Industrial Informatics, 14(7): 3087-3096.

Patel, N. and Borkar, A. (2017). Hybrid control design for swing up and stabilization of cart pendulum system. Paper presented at International Conference on Intelligent Computing, Instrumentation and Control Technologies (ICICICT2017), Kannur, India, 1051-1057, USA: IEEE.

Perruquetti, W. and Barbot, J. P. (2002). Sliding Mode Control in Engineering. CRC Press, Boca Raton, UK.

Shtessel, Y.; C. Edwards; L. Fridman and A. Levant. (2014). Sliding Mode Control and Observation. Birkhäuser Basel, New York, USA.

Siradjuddin, I.; Z. Amalia; B. Setiawan; R. P. Wicaksono and E. Yudaningtyas. (2017). Stabilising A Cart Inverted Pendulum System Using Pole Placement Control Method. Paper presented at 15th International Conference on Quality in Research: International Symposium on Electrical and Computer Engineering, Nusa Dua, Indonesia, 197-203, USA: IEEE.

Siradjuddin, I.; Z. Amalia; B. Setiawan; F. Ronilaya E. Rohadi; A. Setiawan; C. Rahmad and S. Adhisuwignjo. (2018). Stabilising a cart inverted pendulum with an augmented PID control scheme. Paper presented at MATEC Web of Conferences, Nusa Dua, Indonesia, 110-117, EDP Sciences
Soon, C. C.; R. Ghazali; H. I. Jafaar and S. Y. S. Hussein. (2017). Sliding Mode Controller Design with Optimized PID Sliding Surface using Particle Swarm Algorithm. Procedia Computer Science, 105(2017): 235-239.

Udhayakumar, K and Lakshmi, P. (2007). Design of Robust Energy Control for Cart-Inverted Pendulum. International Journal of Engineering and Technology, 4(1): 66-76.

Urniežius, R. and Gegužis, E. (2014). Hybrid fuzzy logic and adaptive LQR controller for swing-up, positioning and stabilization of inverted pendulum. Elektronika ir Elektrotechnika, 20(3): 11-15.

Wang, G.; K. Zheng; X. Wang and S. Yu. (2013). Sliding Mode Control Design for a Class of SISO Systems with Uncertain Sliding Surface. Mathematical Problems in Engineering, 2013(1): 1-7.

Yih, C. C. (2013). Sliding Mode Control for Swing-Up and Stabilization of the Cart-Pole Underactuated System. Asian Journal of Control, 15(4): 1201-1214.

Yusuf, L. A. and Magaji, N. (2014). Performance Comparison of Position Control of Inverted Pendulum Using PID and Fuzzy Logic Controllers. Bayero Journal of Engineering and Technology (BJET), 9(2): 48-55.

Zhang, J.; P. Shi; Y. Xia and H. Yang. (2019). DiscreteTime Sliding Mode Control with Disturbance Rejection. IEEE Transactions on Industrial Electronics, 66(10): 7967-7975. 